\title{
Finding Division Points for Time-Series Corpus Based on Topic Changes
}

\author{
Hiroshi Kobayashi and Ryosuke Saga \\ Osaka Prefecture University, 1-1, Gakuen-cho, Naka-ku, Sakai-shi, Osaka, Japan \\ kobayahi@mis.cs.osakafu-u.ac.jp, saga@cs.osakafu-u.ac.jp
}

\begin{abstract}
This paper describes the discovery method of finding proper points for dividing a corpus with time series information for extracting local and frequent keywords. Local and frequent keywords express a corpus with time series information and are useful for comprehending it. To extract keywords from the corpus, the previous works proposed corpus separating method. However, this method divides the corpus at equal intervals so that it cannot take into account the change of topic. To consider the change of topics and divide the corpus based on it, we utilize the idea of topic model and the topic extracted by Latent Dirichlet Allocation (LDA). In the experiment using newspaper articles during five years topics, we confirm that the topics of each document change as time passed by using the output from LDA and the point which is available on dividing the corpus by the change of topics notably is observable.
\end{abstract}

Keywords: keyword extraction, time series information, LDA.

\section{Introduction}

In recent years, many documents have been converted to digital form by the development of the Internet. This transition to "paperless" documentation reduces the time and effort required to manage documents and makes necessary information instantly available. The amount of digital document data is increasing enormously. Such a large amount of document data may be collectively saved as a corpus. A corpus aggregates a considerable amount of digitalized document data and is used for research such as natural-language processing [1]. To efficiently acquire information from a corpus, the features of the corpus must be known.

Keyword extraction is one way to effectively learn the features of a corpus [2]. Keywords and keyword extraction make searching and summarizing a corpus easy [3]. For example, when searching a document, the keyword expressing the document's content is useful to filter your results [4]. The keywords are first ranked by importance, and the top $N$ keywords are extracted. The frequency of a word in the whole corpus is conventionally used as a measure of importance. Based on the frequency, there are several proposed ranking methods such as term frequency and term frequency-inverse document frequency (TF-IDF).

In contrast, several types of corpuses have time series, such as newspaper articles, magazines, papers, and blogs. Corpuses also include locally characteristic keywords, 
which appear locally and frequently. These keywords are important to comprehend trends and situations. For example, these keywords appear on Twitter as Trends. For extracting keywords from a corpus with time-series information, conventional methods based on word frequency are not very effective because the keywords are compared with the whole corpus. Therefore, the words which appear frequently and locally are not extracted because the weights from the methods tend to become low. But, when such a word also expresses a corpus, the word which appears frequently locally isn't extracted because the weight becomes low.

To solve this problem, Saga et al. proposed a method of dividing a corpus [5]. The words that appear locally can be extracted as keywords from the divided corpus because division narrows the period to observe documents. However, although normally the change of topic should be considered, because these keywords express the trend of topics, a corpus is divided at equal intervals in this method. Therefore, we should divide the corpus while considering changes of topics.

This paper proposes a method to find division points of a corpus with time-series information while also accounting for changes of topics. To comprehend a topic, we utilize the idea of a topic model and identify the topics using Latent Dirichlet Allocation (LDA). The paper is organized as follows. In Section 2, we introduce a conventional keyword extraction method for when a corpus includes time-series information and identify this method's problem. In Section 3, we describe a topic model and LDA. In Section 4 we perform an experiment for news articles to divide corpus by presuming the distribution of a topic in each document using LDA. Finally, Section 5 contains our conclusions and plans for future work.

\section{Keyword Extraction Methods for Corpus with Time-Series Information}

The keyword extraction method calculates the weight of all the words appearing in each document of a corpus. Then, words with large weights from each document are extracted as keywords for the document, and words with large weights across many documents are extracted as keywords for the corpus. The flow is shown in Fig.1. Words are generally assigned weights using TF-IDF and residual IDF [6].

However, for some corpuses, time information is important. For example, newspaper articles and blogs are published and updated daily or weekly, and conference proceedings and annual reports are published annually. These documents are organized in time series and change continuously during a certain period. This paper treats such a time-series corpus.

In a time-series corpus, some words occur frequently only during a specific period of time on account of certain occurrences and incidents. As shown in Fig. 2, when a region receives record amounts of snowfalls out of season, the phrase "heavy snow" appears in newspapers frequently and locally. In this situation, the phrase can be an important keyword (phrase). However, the frequency of the phrase is low from the viewpoint of the whole corpus because many people lose interest after the event. 


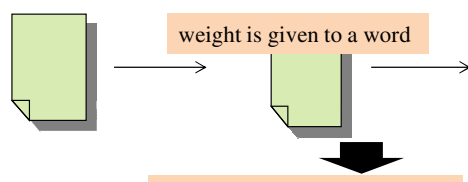

Counting the number of times that the word was extracted as

a keyword of a document .

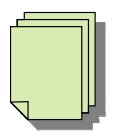

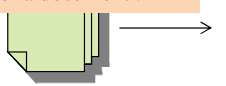

the word with large

weight is extracted as a

keyword of a document

A word with much number of times serves as a keyword of all documents

Fig. 1. The flow of keyword extraction

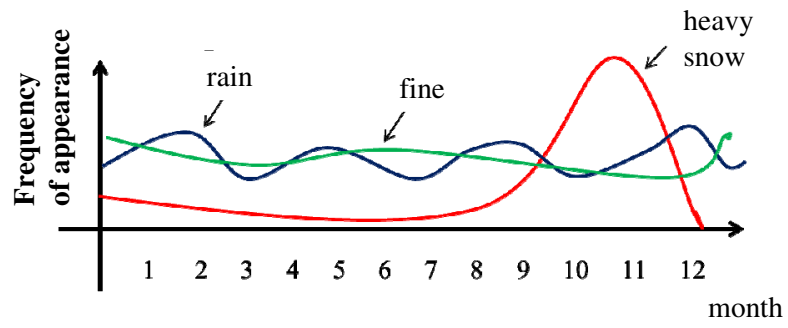

Fig. 2. The word which occurs frequently locally

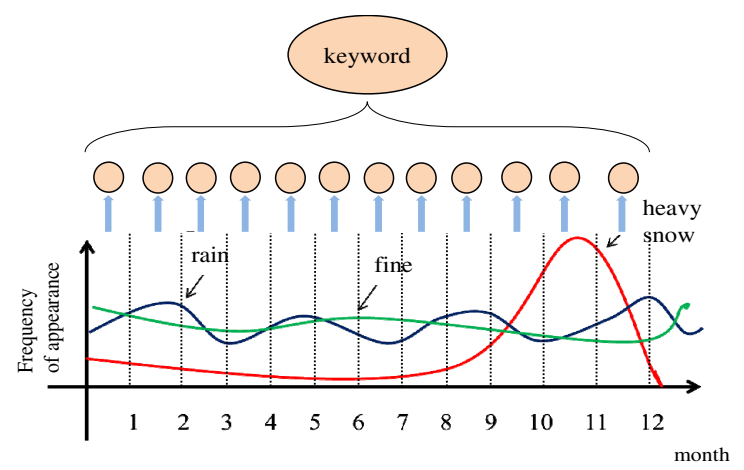

Fig. 3. The aspect of division of a corpus

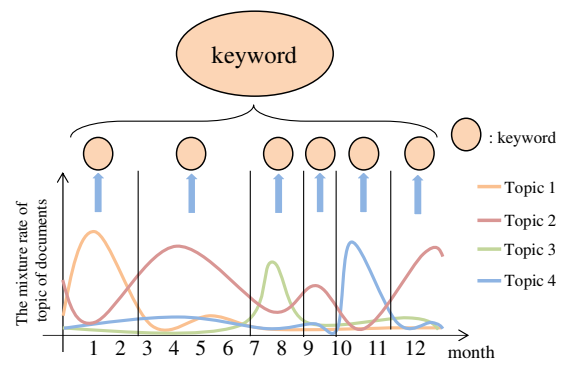

Fig. 4. The aspect of division of a corpus based on the change of topics 
So the weight of the word may be lower than those of the words appearing in only a few documents during that period, but constantly, such as "fine" and "rain". As a result, words that express the features of a corpus like "heavy snow" are often not extracted as keywords.

To solve the problem of local keywords not being correctly extracted, Saga et al. proposed a method of separating a corpus according to time series. If a corpus is separated by this method, even conventional methods can be used to extract words that appear frequently but locally. This method is based on the idea that extraction of words appearing frequently and locally is attained by narrowing the period of observation for documents within a time series. Fig. 3 shows how to apply this approach to the corpus from Fig. 2. As shown in the figure, when the corpus is divided, the phrase "heavy snow," which frequently appears between October and November, can be extracted as a keyword without the interruption of other words.

This approach is useful for real data. Previous studies show how the approach can extract local keywords from newspaper articles. However, the approach divides the corpus at equal intervals. In fact, local keywords express the trends of the occurrence during certain periods, but previous studies ignore these trends.

Therefore, we must find suitable compartmental points to extract characteristic words based on changes of topics. Fig. 4 shows how to divide a corpus of documents based on changes of topics. To observe identify the changes of topics, we can utilize use the idea of topic model [7] and the topics of documents are presumed by use Latent Dirichlet Allocation (LDA) to infer the topics of documents. Using a topic model and LDA, we can try to find suitable points for compartmentalization.

\section{Corpus Separation Approach Based on Topic Change.}

\subsection{Topic Model and LDA (Latent Dirichlet Allocation)}

A topic model is the probable generation model of a document. The probability that a word will appear varies based on document topics, and then, the probabilistic of appearance of a word depends on topic and each document has multiple-topics. This A topic model is also a statistical model for discovering a certain topic from a document. Each document of a document set is classified into topics. Topics show the contents briefly and make searching and classifying documents easy. One document may have multiple topics. This image is shown in Fig. 5. In this figure, document A is categorized into Topic a and Topic b, and the mixture ratios of two topics are 0.6 and 0.4 for each.

The LDA model is a kind type of the language probabilistic model which assumes that a document consists of multiple-topics. A word can be classified into topics and a document can be classified for every topic. Prior distribution is assumed to be Dirichlet distribution. It is because multinomial distribution can be assumed to how to choose words and topics. The graphical model of LDA is shown in Fig.6. The box expresses the number of times of execution. The nodes within box $D$ correspond to the number of times of execution in the document. $\mathrm{N}$ is the number of words and $\mathrm{T}$ is number of topics. Each topic is defined based on the distribution of words by node $\varphi$. Then, it is probability distributions. The distribution of topics in each document is generated by node $\theta$. The topic is randomly selected from the distribution of topics by 
node $\mathrm{Z}$. The word is randomly selected from the distribution of words by node W. When $\mathrm{W}$ is observed, the posterior probability of $\{\varphi, \theta, \mathrm{Z}\}$ is calculated based on probability density approximated by sampling. Then the posterior probability is calculated based on prior probability and the likelihood of $\mathrm{W}$ calculated from a generation model. $\alpha, \beta$ is the parameters to assume that the prior distribution is the Dirichlet distribution and is called the hyper-parameter.

The change of topic is observable because of the distribution of topics obtained by LDA.

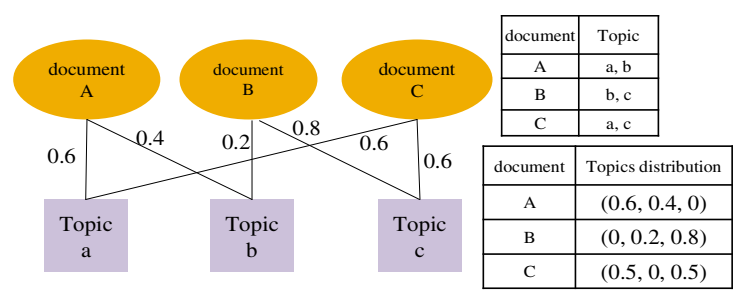

Fig. 5. The image of a multiplex topic

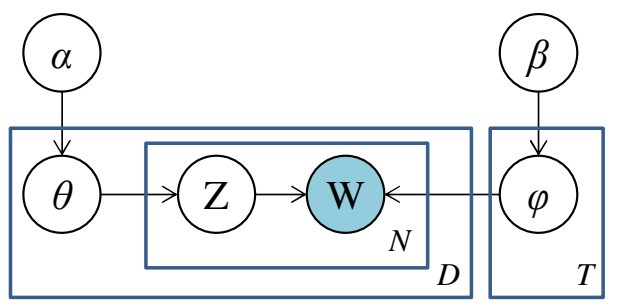

Fig. 6. Graphical model of LDA

\subsection{Process of Separating a Corpus Based on Topic Change by LDA}

The number of topics and hyper-parameters must be set before applying LDA to a corpus. Then, LDA learns by inputting document and outputs the topic distribution of each document. The distribution shows the mixture ratio of the topic which the document contains. Then, by illustrating the mixture ratio using time series, the rate changes of each topic in each document can be identified. These changes are easier to identify in graphs. A corpus is divided at the points where large changes of topics occur.

\section{$4 \quad$ Experiments}

\subsection{Goal and Dataset}

We performed an experiment to find the points where the topics of documents significantly changed. We used 1827 news articles from CNN.com between January 1, 2000 and December 31, 2004 as a time-series document set and the MALLET [9], a Java toolkit for machine-learning applied to natural language, to create the LDA model. 
We set the number of topics (T) to 20, and optimized the hyper-parameters based on the number of topics.

\subsection{Experimental Result}

The word distribution outputted by LDA is shown in Table 1. We could guess some contents of topics from the LDA result. For example, Topic 6 in Table 1 is presumed to be the Iraq War because we can see words such as "iraq," "war," and "baghdad." The mixture ratio of topics in each document is shown in Table 2. We found the changes of topics of a document set, including time-series information, by observing the mixture ratio of a document for every topic. Based on Table 2, we expressed the changes of topics as the line graph shown in Fig.7.

Table 1. The word distributions of 20 topics

\begin{tabular}{|c|c|c|c|c|c|c|c|c|c|}
\hline Topic 1 & Topic 2 & Topic 3 & Topic 4 & Topic 5 & Topic 6 & Topic 7 & Topic 8 & Topic 9 & Topic 10 \\
\hline john & gore & police & president & male & iraq & plane & afghanistan & today & class \\
\hline president & today & area & house & ph & iraqi & space & taliban & percent & width \\
\hline kerry & news & case & white & great & war & flight & bin & news & style \\
\hline campaign & percent & county & clinton & big & baghdad & china & laden & $\operatorname{tax}$ & john \\
\hline senator & mocain & question & senator & thing & saddam & air & united & president & stewart \\
\hline election & online & shooting & blitzer & break & forces & crew & war & press & $\mathrm{src}$ \\
\hline vote & government & morning & george & news & military & question & president & question & table \\
\hline voters & correspondent & sniper & day & commercial & hussein & chinese & states & economy & href \\
\hline george & elian & investigation & united & novak & troops & today & al & market & height \\
\hline state & anchor & washington & country & lin & city & aircraft & military & cut & option \\
\hline debate & located & point & john & female & coalition & day & today & online & brien \\
\hline tonight & fdch & scene & american & story & air & miles & terrorism & anchor & color \\
\hline presidential & secure & person & republican & love & today & united & qaeda & house & today \\
\hline gore & clinton & man & bill & camera & iraqis & states & york & fdch & dean \\
\hline democratic & york & law & state & three & general & morning & osama & case & martha \\
\hline states & states & shot & party & coming & soldiers & officials & country & kagan & border \\
\hline candidate & case & enforcement & states & day & marines & point & security & bill & type \\
\hline night & bill & chief & great & brien & question & course & pakistan & located & input \\
\hline $\mathrm{al}$ & day & virginia & senate & long & army & airport & government & correspondent & align \\
\hline Topic 11 & Topic 12 & Topic 13 & Topic 14 & Topic 15 & Topic 16 & Topic 17 & Topic 18 & Topic 19 & Topic 20 \\
\hline palestinian & storm & anthrax & iraq & material & cnnbodytext & case & court & condit & iraq \\
\hline israeli & hurricane & enron & war & user & class & today & florida & levy & today \\
\hline israel & florida & question & weapons & news & iraq & question & gore & police & kerry \\
\hline arafat & hour & $\mathrm{fbi}$ & united & copyright & case & court & supreme & news & morning \\
\hline palestinians & area & government & saddam & network & media & morning & election & congressman & president \\
\hline peace & winds & president & cnnbodytext & license & fdch & death & state & chandra & brien \\
\hline israelis & water & news & class & cable & today & family & votes & today & costello \\
\hline east & miles & today & hussein & purposes & california & trial & ballots & gary & security \\
\hline middle & morning & house & president & redistribute & schwarzenegger & jury & county & question & american \\
\hline yasser & power & security & inspectors & rights & brien & police & vote & case & hemmer \\
\hline sharon & damage & war & nations & house & news & judge & campaign & washington & break \\
\hline minister & coming & states & council & media & dean & attorney & today & online & al \\
\hline jerusalem & coast & september & north & long & court & neville & recount & located & iraqi \\
\hline violence & city & united & security & high & question & child & case & correspondent & live \\
\hline president & beach & point & states & specific & defense & evidence & count & secure & intelligence \\
\hline prime & hit & pakistan & korea & federal & cooper & $\operatorname{man}$ & judge & fdch & war \\
\hline state & hours & fact & resolution & provide & state & defense & president & anchor & government \\
\hline secretary & center & intelligence & iraqi & interests & morning & harris & law & point & day \\
\hline united & live & mail & today & prepared & governor & fire & vice & family & case \\
\hline
\end{tabular}


From Fig. 7, we confirmed that periods existed in which the topics of documents changed notably. Topics changed significantly when events related to the topics occurred, and there are related keywords about the topics in each period. Thus, we found clues about suitable separation points for changes of topics.

However, some topics, such as topic 2 and topic 10, could not be guessed from words, whereas some topics, such as Topic 5, remained at a steady state throughout the test period, i.e., we could not find any significant changes of topic. We can guess several reasons for the appearance of such a topic. For example, the number of topics may be wrong for LDA, or LDA may not be able to extract proper topics naturally from a corpus with a time series. Regardless of the reason, some topics extracted from LDA change significantly and some slightly, so we must identify and filter useful topics to discover separation points.

Table 2. Part of the topic distribution of documents

\begin{tabular}{|r|c|c|c|c|c|c|}
\hline document & topic & $\begin{array}{l}\text { The mixture ratio } \\
\text { ofthe topic }\end{array}$ & topic & $\begin{array}{l}\text { The mixture ratio } \\
\text { of the topic }\end{array}$ & topic & $\begin{array}{l}\text { The mixture ratio } \\
\text { of the topic }\end{array}$ \\
\hline $2000 / 1 / 1$ & 4 & 0.428 & 6 & 0.207 & 1 & 0.178 \\
\hline $2000 / 1 / 2$ & 4 & 0.339 & 1 & 0.337 & 6 & 0.113 \\
\hline $2000 / 1 / 3$ & 4 & 0.377 & 1 & 0.367 & 3 & 0.108 \\
\hline $2000 / 1 / 4$ & 1 & 0.467 & 4 & 0.226 & 3 & 0.131 \\
\hline
\end{tabular}

:

\begin{tabular}{|c|c|c|c|c|c|c|}
\hline $2004 / 12 / 28$ & 11 & 0.581 & 4 & 0.144 & 19 & 0.132 \\
\hline $2004 / 12 / 29$ & 11 & 0.598 & 4 & 0.110 & 19 & 0.095 \\
\hline $2004 / 12 / 30$ & 11 & 0.542 & 19 & 0.132 & 4 & 0.126 \\
\hline $2004 / 12 / 31$ & 11 & 0.365 & 4 & 0.246 & 19 & 0.137 \\
\hline
\end{tabular}

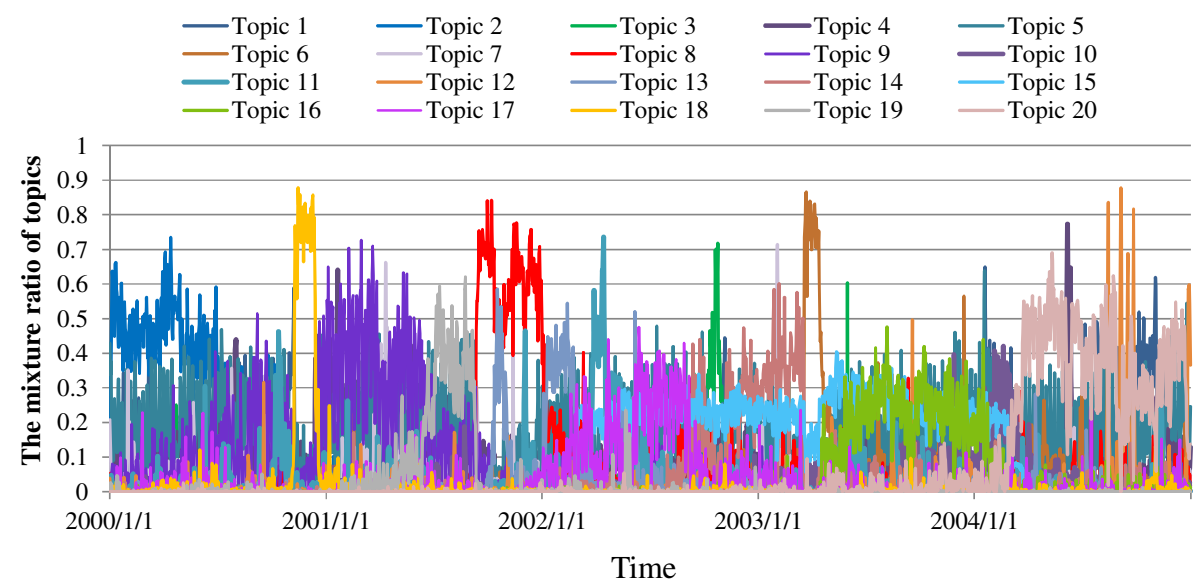

Fig. 7. The change of the mixture ratio of a document 


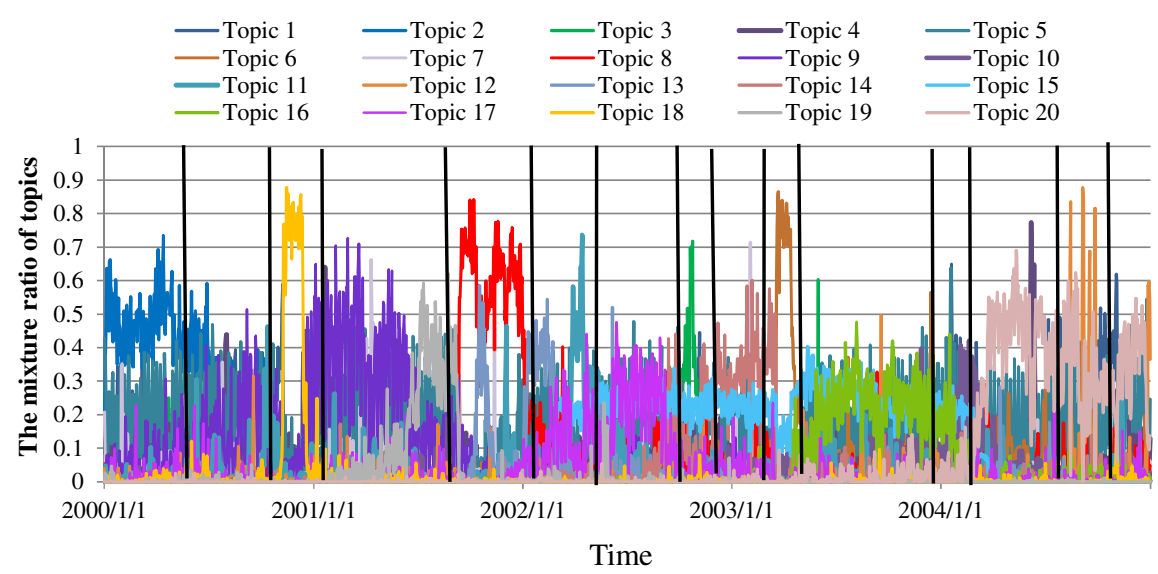

Fig. 8. The image of dividing a time series document set

\section{Conclusion}

In this paper, we proposed a method for finding suitable points of a corpus that includes time-series information to extract-as keywords - characteristic words appearing locally. In this case, dividing a corpus into equal time intervals is insufficient. Therefore, we needed to identify suitable compartments into which the corpus could be divided. The characteristic words appearing in a period, which is related to the topic of the document, change significantly over time. Therefore, we used LDA to examine changes of topics. As a result, we noticed that the main topics of documents changed as time passed. We can expect to extract characteristic words as keywords by dividing a document group at those points where the topic changes significantly. Fig. 8 illustrates the division of a time-series document set.

In this paper, when we performed LDA, we manually set the number of topics to 20. However, the optimal number of topics is unknown. Therefore, in future work we aim to automate the process of choosing the number of topics and evaluate the method of keyword extraction for a time-series document set

Acknowledgement. This research was supported by The Ministry of Education, Culture, Sports, Science and Technology (MEXT), Japan Society for the Promotion of Science(JSPS), Grant-in-Aid for Young Scientists (B), 23760358 and Grant-in-Aid for Scientific Research (C), 25420448.

\section{References}

1. Liu, V., Curran, R.: Web Text Corpus for Natural Language Processing. In: Proceedings of the 11th Conference of The European Chapter of The Association for Computational Linguistics, Trento, Italy, pp. 233-240 (2006) 
2. Liu, F., Liu, F., Liu, Y.: Automatic Keyword Extraction for TheMeeting Corpus Using Supervised Approach and Bigram Expansion. In: IEEE Workshop on Spoken Language Technology, pp. 181-184 (2008)

3. Dredze, M., Wallach, H., Puller, D., Pereira, F.: Generating Summary Keywords for Emails UsingTopics. In: Proceedings of The 2008 International Conference on Intelligent User Interfaces, pp. 199-206 (2008)

4. Litvak, M., Last, M.: Graph-based Keyword Extraction for Single-Document Summarization. In: Proceeding of The Workshop on Multi-source Multilingual Information Extraction and Summarization, pp. 17-24. Association for Computational Linguistics (2008)

5. Saga, R., Tsuji, H.: Improved Keyword Extraction by Separation into Multiple Document Sets According to Time Series. In: HCII, CCIS 374, pp. 450-453 (2013)

6. Church, K., Gale, W.: Inverse Document Frequency (IDF): A Measure of Deviations from Poisson. In: Proceedings of the Third Workshop on Very Large Corpora, pp. 121-130 (1995)

7. Blei, D.M., Ng, A.Y., Jordan, M.I.: Latent Dirichlet Allocation. Journal of Machine Learning Research 3, 993-1022 (2003)

8. McCallum, K.A.: MALLET, http://mallet.cs.umass . edu 